\title{
Assessment of the diagnostic accuracy of home blood pressure monitoring by patients
}

\author{
Sepideh Hajian ${ }^{\circledR}$, Nafiseh Rastgoo $^{2^{*}}$, Sanaz Jamshidi ${ }^{\circledR}$ \\ ${ }^{1}$ Department of Nephrology, Velayat Hospital, Qazvin University of Medical Sciences, Qazvin, Iran \\ ${ }^{2}$ Metabolic Diseases Research Center, Research Institute for Prevention of Non-Communicable Diseases, Qazvin University of Medical \\ Sciences, Qazvin, Iran \\ ${ }^{3}$ Qazvin University of Medical Sciences, Qazvin, Iran
}

\section{A R T I C L E I N F O}

Article Type:

Original

\section{Article History:}

Received: 2 July 2020

Accepted: 5 October 2020

Published online: 29 October 2020

\section{Keywords:}

Hypertension, Out-of-clinic

blood pressure measurement,

Home blood pressure

monitoring (HBPM), 24-

Hour ambulatory blood

pressure monitoring (ABPM),

Diagnostic accuracy

\begin{abstract}
A B S T R A C T
Introduction: According to available guidelines, home blood pressure monitoring (HBPM) can be used to diagnose hypertension and monitor its treatment; however, its effectiveness has rarely been studied in developing countries, including Iran.

Objectives: This study aimed to evaluate the diagnostic accuracy of HBPM, as compared with that of 24-hour ambulatory blood pressure monitoring (ABPM) and office blood pressure measurement (OBPM).

Patients and Methods: This study was conducted on 28 patients suspected of having primary hypertension. The blood pressure of the patients was measured by four methods. Initially, blood pressure was measured by a non-physician using a digital sphygmomanometer in a clinic (OBPM-Digital). After about 1 hour, blood pressure was measured by a physician at the clinic using a mercury sphygmomanometer (OBPM-Mercury). In the third stage, the patient's blood pressure was monitored for 24 hours by the ABPM method. In the fourth stage, each subject used a digital sphygmomanometer to measure HBPM for seven consecutive days.

Results: The blood pressure values measured through the ABPM method were significantly lower than those measured by other methods $(P<0.05)$. The prevalence of hypertension diagnosed by OBPM-Mercury, OBPM-Digital, HBPM, and ABPM method was $82 \%$, 54\%, $50 \%$, and $21 \%$, respectively. As compared with $\mathrm{ABPM}$ as the gold standard, the diagnostic accuracy of HBPM, OBPM-Digital, and OBPM-Mercury was $64 \%, 61 \%$, and $32 \%$, respectively. The frequency of white coat hypertension $(\mathrm{WCH})$ diagnosed by HBPM and ABPM methods was $39 \%$ and $64 \%$, respectively, and the frequency of masked hypertension $(\mathrm{MH})$ diagnosed was $7 \%$ and $4 \%$, respectively. The sensitivity, specificity, and diagnostic accuracy of HBPM, as compared with ABPM, in detecting $\mathrm{MH}$ were $100 \%, 96 \%$, and $97 \%$, respectively; in addition, as compared with $\mathrm{WCH}$, they were $56 \%, 90 \%$, and $68 \%$, respectively.

Conclusion: The findings of the present study showed that HBPM had higher diagnostic accuracy than OBPM in diagnosing hypertension. Also, HBPM was able to detect MH with a high level of diagnostic accuracy, and in more than two-thirds of cases, it was also able to detect WCH and diagnose patients with sustained hypertension.
\end{abstract}

Implication for health policy/practice/research/medical education:

Our study showed that home blood pressure monitoring can be used with high diagnostic accuracy in hypertension detection, determining its phenotypes, and monitoring its treatment.

Please cite this paper as: Hajian S, Rastgoo N, Jamshidi S. Assessment of the diagnostic accuracy of home blood pressure monitoring by patients. J Renal Inj Prev. 2021; 10(4): e31. doi: 10.34172/jrip.2021.31.

\section{Introduction}

Hypertension is one of the most important and wellknown risk factors for cardiovascular diseases, chronic kidney disease, and cerebrovascular accidents. Based on World Health Organization (WHO) estimates, high blood pressure is $25 \%$ prevalent among the world's population and is projected to reach $60 \%$ by 2025 (1).

Although office blood pressure measurement (OBPM) is primarily the usual method of diagnosing and treating hypertension, there may be significant differences between OBPM and out-of-clinic blood pressure measurements (2). There are two common methods of out-of-clinic 
blood pressure measurements, including automatic and outpatient ambulatory 24-hour blood pressure monitoring (ABPM) and home blood pressure monitoring (HBPM). As the main advantage of measuring blood pressure outside a clinic, it provides more natural and real values for patients' blood pressure, when they are away from a clinic environment. The use of these methods has led to the detection of phenotypes in patients with hypertension, including white coat hypertension (WCH) and masked hypertension $(\mathrm{MH})$ (3). WCH occurs when a patient is diagnosed with hypertension when undergoing OBPM, though he/she has normal blood pressure outside a clinic. Conversely, when a patient's blood pressure is normal in the clinic but is high outside the clinic, this condition is called $\mathrm{MH}$. These two phenotypes are very common in both treated and untreated patients, and it is not possible to detect them when measurements are performed only in a clinic $(3,4)$.

It is of great importance to recognize hypertensive phenotypes because $\mathrm{WCH}$ is associated with a slight or no increase in cardiovascular events and mortality, as compared with normotensive people (5). In contrast, uncontrolled $\mathrm{MH}$ is associated with increased cardiovascular events and mortality (6). In practice, however, patients with $\mathrm{WCH}$ who are often treated are exposed to multiple side effects of drugs; besides, the disease can also impose high costs on patients and the health care system due to the widespread prevalence of hypertension. On the other hand, patients with $\mathrm{MH}$ who need to be treated are often undiagnosed and therefore, not treated. In general, the prevalence of $\mathrm{WCH}$ is estimated to be $40-30 \%$, and the prevalence of $\mathrm{MH}$ is estimated to be 10\%-30\% (7).

Although ABPM is considered to be the gold standard for measuring blood pressure (8), its use is associated with a high cost. Moreover, ABPM is not available to all patients and in all centers. It is a good method for measuring blood pressure in certain cases, but it is not good for long-term monitoring of blood pressure. HBPM, on the other hand, allows the patient to measure and monitor blood pressure at every desired time and is associated with a high level of patient acceptance. In addition, the use of HBPM for monitoring patients undergoing treatment is associated with better control of blood pressure (9). However, it is necessary to train patients to use this method properly (10).

\section{Objectives}

Common guidelines for measuring blood pressure recommend using $\mathrm{ABPM}$ and $\mathrm{HBPM}$ to diagnose patients and monitor the process of treatment (7). However, the effectiveness of this method has rarely been studied in lowincome and developing countries, including Iran. Also, the rate of the acceptance of blood pressure monitoring methods may vary among different races; hence, it is necessary to consider clinical needs, patients' preferences, and cultural barriers in choosing an appropriate method for monitoring high blood pressure (11). Therefore, this study aimed to determine the diagnostic accuracy of HBPM, as compared with that of ABPM (as the gold standard method of measuring blood pressure), and OBPM.

\section{Patients and Methods \\ Study design}

This study was conducted on patients over 18 years of age suspected of having hypertension, which referred to a clinic. They either had a blood pressure of above 135/85 $\mathrm{mm} \mathrm{Hg}$ at home or blood pressure of above 140/90 mm Hg in an office, or as diagnosed by a physician, had symptoms of hypertension, and needed further examination. Exclusion criteria were chronic hypertension, secondary hypertension, and unwillingness to participate in the study. The patients without access to the necessary items required for assessing blood pressure at home were excluded from the study. A total of 28 patients were non-randomly selected based on the order of admission. Written consent was obtained from all the patients.

After explaining the objectives and the method of measurements to each patient, the data on age, gender, weight, and height of the patients were recorded using a form. Then, the blood pressure of the patients was measured by four methods. First, using a digital sphygmomanometer and under appropriate conditions, systolic and diastolic blood pressures of the patients were measured twice by a non-physician by Omron digital sphygmomanometer (model: M3 HEM-7131-E, Omron healthcare Co., Japan), and the mean values were recorded as the values obtained from digital clinic blood pressure measurement (OBPM-Digital). Then, after about an hour, systolic and diastolic blood pressures of the patients were measured twice by a physician using a mercury sphygmomanometer. The mean values were recorded as office blood pressure measurements with a mercury sphygmomanometer (OBPM-Mercury).

In the third stage, using an ABPM device (Cardio Pro ABPM System device, ACT Co., Iran), the blood pressure of the patients was measured for 24 hours. The mean systolic and diastolic blood pressures during the phase of awakening, throughout the day, and during sleep time were measured every 20 minutes, and the total mean systolic and diastolic blood pressures were recorded. During the sleep phase, a 10\%-20\% reduction of systolic or diastolic blood pressure was considered as dipping, and an increase was considered as reverse hypertension. Moreover, if blood pressure was higher than 130/85 mmHg immediately after waking up, it was considered an increase in morning blood pressure (morning surge). The mean heart rate was also measured by ABPM. In the fourth stage, patients were trained to perform HBPM by Omron 
digital sphygmomanometer (Model: M3 HEM-7131-E, Omron healthcare Co., Japan). To assess blood pressure at home, each patient was required to measure his/her blood pressure at home twice a day for seven consecutive days in the morning and evening and recorded it in a chart. When reviewing the charts, the measurements performed on the first day were excluded from the calculation, and the mean value of measurements performed in the next six days was considered the patient's blood pressure measured at home.

Systolic blood pressure $\geq 140 \mathrm{~mm} \mathrm{Hg}$ and diastolic blood pressure $\geq 90 \mathrm{~mm} \mathrm{Hg}$ measured by OBPM (digital or mercury), systolic blood pressure $\geq 130 \mathrm{~mm} \mathrm{Hg}$, and diastolic blood pressure $\geq 80 \mathrm{~mm} \mathrm{Hg}$ measured by $\mathrm{ABPM}$, and systolic blood pressure $\geq 135 \mathrm{~mm} \mathrm{Hg}$ and diastolic blood pressure $\geq 85 \mathrm{mmHg}$ measured by HBPM were considered as hypertension. Besides, the presence of systolic or diastolic hypertension in each method was considered as the presence of hypertension in that method (7).

The phenotype of patients' blood pressure status was determined in OBPM measurements and compared with HBPM and ABPM. If the patient's blood pressure was normal in both methods, it was considered as a normotensive condition, and if hypertension was observed in both methods, it was considered as sustained hypertension. WCH was considered when the first method showed hypertension, and the second method indicated normal blood pressure. In the opposite case, the condition was considered as $\mathrm{MH}$.

\section{Statistical analysis}

SPSS 25 statistical software was used for data entry and analysis. Frequency (percentage) was used to describe variables, and a paired $t$ test was used to compare blood pressures measured by different methods. ABPM was considered the gold standard, and the diagnostic efficiency (sensitivity, specificity, positive predictive value, negative predictive value, and diagnostic accuracy) of other methods was calculated. The diagnostic efficiency of HBPM in detecting each hypertensive phenotype was also assessed, as compared with ABPM. A $P<0.05$ was set as the level of significance.

\section{Results}

Of all the participants, 18 patients (64\%) were female, and ten people $(36 \%)$ were male. The mean $(\mathrm{SD})$ of the patients' age was $49 \pm 10$ years. The mean (SD) of peoples' height, weight, and body mass index (BMI) were $167 \pm 11$ $\mathrm{cm}, 75 \pm 12 \mathrm{~kg}$, and $27.1 \pm 4.7 \mathrm{~kg} / \mathrm{m}^{2}$, respectively.

Table 1 and Figure 1 show the mean systolic and diastolic blood pressure measured by different methods, and they are compared with the values measured by $\mathrm{ABPM}$. The mean (SD) of heart rate recorded by ABPM was $76 \pm 10$ beats per minute. In addition, 21 people (75\%) had hypotension during the sleep phase, and 14 people
(50\%) had morning hypertension. The highest levels of blood pressure were observed in cases measured by OBPM-Mercury. The values of blood pressure measured by OBPM-Digital, OBPM-Mercury, and HBPM were significantly higher than the values measured through ABPM $(P<0.05)$.

Table 2 and Figure 2 show the frequency of hypertension in different methods based on criteria defined in the methods section. The prevalence of hypertension had a decreasing trend in cases monitored by OBPM-Mercury, OBPM-Digital, HBPM, and ABPM methods, respectively. The frequency of hypertension measured by the OBPMMercury method was almost four times. The frequency of hypertension measured by OBPM-Digital and HBPM was more than twice higher than the frequency of hypertension measured by the ABPM method.

Table 3 shows the diagnostic efficiency (sensitivity, specificity, positive predictive value, negative predictive value, and diagnostic accuracy) of different methods of measuring blood pressure compared to ABPM as the gold standard. HBPM had the highest diagnostic accuracy, while OBPM-Mercury blood pressure measurement had

Table 1. Mean systolic and diastolic blood pressure measured in different methods

\begin{tabular}{llcc}
\hline Method & & Mean (SD) & P value* \\
\hline \multirow{2}{*}{ ABPM } & Systolic, mm Hg & $121 \pm 19$ & - \\
& Diastolic, mm Hg & $72 \pm 13$ & - \\
\multirow{2}{*}{ OBPM-Mercury } & Systolic, mm Hg & $143 \pm 19$ & $<0.001$ \\
& Diastolic, mm Hg & $91 \pm 15$ & $<0.001$ \\
OBPM-Digital & Systolic, mm Hg & $135 \pm 24$ & 0.001 \\
& Diastolic, mm Hg & $84 \pm 15$ & $<0.001$ \\
HBPM & Systolic, $\mathrm{mm} \mathrm{Hg}$ & $130 \pm 16$ & 0.005 \\
& Diastolic, $\mathrm{mm} \mathrm{Hg}$ & $83 \pm 8$ & $<0.001$ \\
\hline
\end{tabular}

* Comparison of different methods with ABPM mean, paired $t$ test. ABPM: Ambulatory blood pressure monitoring, OBPM-Mercury: office blood pressure measurement with mercury sphygmomanometer, OBPM-Digital: office blood pressure measurement with digital sphygmomanometer, HBPM: home blood pressure monitoring.

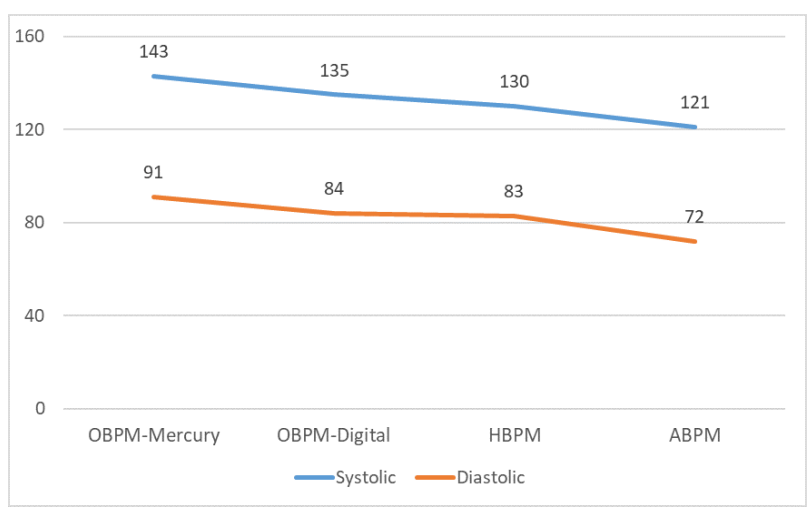

Figure 1. Mean systolic and diastolic blood pressure in different methods. 
Table 2. Frequency of hypertension in various measurement methods*

\begin{tabular}{llc}
\hline Method & & Hypertension, No (\%) (n=28) \\
\hline \multirow{3}{*}{ ABPM } & Systolic & $6(21 \%)$ \\
& Diastolic & $6(21 \%)$ \\
& Overall & $6(21 \%)$ \\
OBPM-Mercury & Systolic & $18(64 \%)$ \\
& Diastolic & $20(71 \%)$ \\
& Overall & $23(82 \%)$ \\
OBPM-Digital & Systolic & $11(39 \%)$ \\
& Diastolic & $13(46 \%)$ \\
& Overall & $15(54 \%)$ \\
HBPM & Systolic & $12(43 \%)$ \\
& Diastolic & $10(36 \%)$ \\
\hline
\end{tabular}

* Definition of hypertension level in each method according to Patients and Methods section.

ABPM: Ambulatory blood pressure monitoring, OBPM-Mercury: office blood pressure measurement with mercury sphygmomanometer, OBPM-Digital: office blood pressure measurement with digital sphygmomanometer, HBPM: home blood pressure monitoring.

the lowest diagnostic accuracy. In all the three methods, the positive predictive value was equal to, or less than $36 \%$, and the negative predictive value was more than $80 \%$.

Table 4 and Figure 3 show the status of OBPMs, compared with those measured by ABPM and HBPM. Table 5 shows the diagnostic efficacy of HBPM over ABPM in detecting hypertensive phenotypes (both are compared with OBPM-Mercury). The results presented in this table indicate that HBPM can detect $\mathrm{MH}$ with high diagnostic accuracy, and it is also able to detect $\mathrm{WCH}$ and diagnose patients with sustained hypertension in more than two-thirds of cases.

\section{Discussion}

The findings of the present study showed that the mean blood pressure measured by ABPM was lower than the values measured through other methods. Following ABPM, HBPM showed the lowest frequency of hypertension and had the highest diagnostic accuracy, as compared with OBPM-Mercury or OBPM-Digital. HBPM was also highly sensitive and more specific than the other two methods. In addition, HBPM was able to detect $\mathrm{MH}$ with high diagnostic accuracy. In more than two-thirds of cases, it was also able to detect $\mathrm{WCH}$ and diagnose patients with sustained hypertension.

Various studies have assessed the diagnostic efficacy of

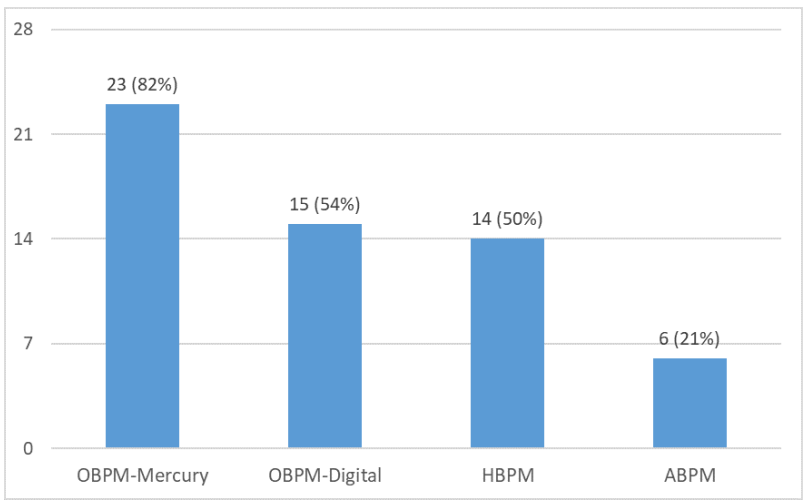

Figure 2. Frequency of hypertension based on different methods.

HBPM versus ABPM in different groups of patients, such as treated or untreated patients, diabetic patients, etc (12). According to the results of a review study by Stergiou and Bliziotis, the sensitivity and specificity of diagnosis of hypertension using HBPM in untreated patients were $48 \%-100 \%$ and $44 \%-93 \%$, respectively. In comparison, in treated patients, they were $52 \%-97 \%$, and is $84-63 \%$, respectively (12). Based on the results of Kang and colleagues' study, HBPM had low sensitivity (47\%-74\%) but high specificity ( $86 \%-95 \%)$, positive predictive value (41\%-87\%), and negative predictive value (82\%-94\%) as compared with ABPM (13). In our study, as compared with ABPM, the sensitivity of all the three methods, including HBPM, OBPM-Mercury, and OBPM-Digital, was the same and equal to $83 \%$. However, the specificity of HBPM was $59 \%$ that was more than three times higher than that of OBPM-Mercury. Overall, HBPM had higher diagnostic accuracy than the two OBPM methods. The negative predictive value in all three methods was higher than $80 \%$, indicating that in observing normal blood pressure in these methods, it can be concluded, with a very high probability, that the patient does not have hypertension. However, considering the positive predictive value of these methods (less than 36\%), when hypertension is detected by these methods, it is necessary to confirm the diagnosis by repeated measurements or using ABPM.

In general, the prevalence of $\mathrm{WCH}$ is $30-40 \%$, and the prevalence of $\mathrm{MH}$ is estimated to be $10 \%-30 \%(7,14)$. In the study by Nasothimiou et al, the prevalence of $\mathrm{WCH}$ in $\mathrm{HBPM}$ and $\mathrm{ABPM}$ was $14 \%$ and $15 \%$, respectively, and accordingly, the prevalence of $\mathrm{MH}$ was $16 \%$ and $15 \%$,

Table 3. Diagnostic efficiency of different blood pressure measurement methods compared to ABPM

\begin{tabular}{|c|c|c|c|c|c|}
\hline Method & Sensitivity & Specificity & Positive predictive value & Negative predictive value & Diagnostic accuracy \\
\hline OBPM-Mercury & $83 \%$ & $18 \%$ & $22 \%$ & $80 \%$ & $32 \%$ \\
\hline OBPM-Digital & $83 \%$ & $55 \%$ & $33 \%$ & $92 \%$ & $61 \%$ \\
\hline HBPM & $83 \%$ & $59 \%$ & $36 \%$ & $93 \%$ & $64 \%$ \\
\hline
\end{tabular}

ABPM: Ambulatory blood pressure monitoring, OBPM-Mercury: office blood pressure measurement with mercury sphygmomanometer, OBPMDigital: office blood pressure measurement with digital sphygmomanometer, HBPM: home blood pressure monitoring. 


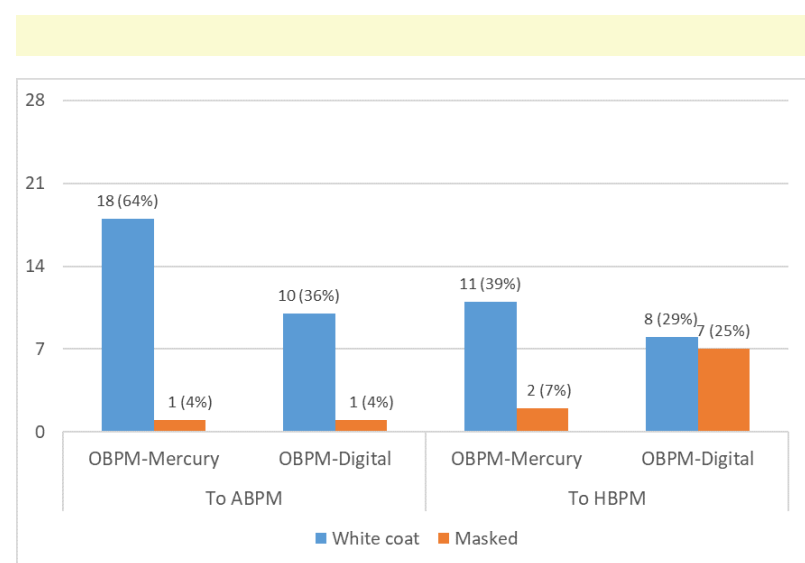

Figure 3. Frequency of hypertension phenotypes (white coat and masked).

respectively (15). In our study, the frequency of $\mathrm{WCH}$ in HBPM and ABPM was 39\% and 64\%, respectively, and the frequency of $\mathrm{MH}$ was $7 \%$ and $4 \%$, respectively. Differences in the prevalence of $\mathrm{MH}$ and $\mathrm{WCH}$ in different centers, and studies can be attributed to differences in sample sizes, characteristics of patients, and study centers.

In Nasothimiou et al study, compared with ABPM, the sensitivity and specificity of HBPM in the diagnosis of sustained hypertension were $90 \%$ and $89 \%$, respectively. While there were $61 \%$ and $94 \%$ in the diagnosis of $\mathrm{WCH}$, respectively, and were $60 \%$ and $93 \%$ in the diagnosis of $\mathrm{MH}$, respectively (15). In our study, compared with ABPM, the sensitivity, specificity, and diagnostic accuracy of HBPM in the diagnosis of $\mathrm{MH}$ were $100 \%, 96 \%$, and $97 \%$, respectively, and in the diagnosis of $\mathrm{WCH}$ were $56 \%, 90 \%$, and $68 \%$, respectively. It indicates that HBPM had high sensitivity and specificity in the diagnosis of $\mathrm{MH}$. However, it has low sensitivity and specificity in the diagnosis of WCH.

Evidence suggests that HBPM or ABPM methods should be used to confirm the diagnosis of hypertension and monitor its long-term process of management. There is strong evidence that the mentioned methods are superior to the routine methods of blood pressure measurement in a clinic and are more effective in predicting the damage in a target organ caused by hypertension and the occurrence of cardiovascular events $(7,16,17)$. As compared with routine methods of blood pressure monitoring, HBPM is associated with an improved level of blood pressure control (18). Besides that, the measurement of blood pressure levels at home are more repeatable than the measurements performed in a clinic and have a better predictive value (19) and may reduce the burden of hypertension (7). However, there is still controversy about whether HBPM can act as an alternative to ABPM or not. In some cases, it has been reported that because of the moderate agreement between the two methods, they may not be considered as alternatives to each other $(13,20)$. Although, in some guidelines, ABPM is mentioned as the preferred method of evaluation (2), it is still considered as an expensive method, unaffordable for some patients, and it can only be used in specialized centers. Therefore, HBPM is considered an important component of hypertension management and is utilized to evaluate and treat hypertension. In the case of the appropriate application of protocols, HBPM can be used instead of ABPM when it is not available (8). Thus, HBPM is increasingly used in many countries (21) and today guidelines, developed in the United States, Canada, and Europe for the management of hypertension emphasized the importance of out-of-clinic blood pressure monitoring for the daily management of patients with hypertension. (7). However, in low- and middle-income

Table 4. Hypertension phenotypes status measured in the clinic compared to outside the clinic

\begin{tabular}{lccc}
\hline & Normotensive & Sustained Hypertension & White coat hypertension \\
\hline OBPM-Mercury to ABPM & $4(14 \%)$ & $5(18 \%)$ & $18(64 \%)$ \\
OBPM-Digital to ABPM & $12(43 \%)$ & $5(18 \%)$ & $10(36 \%)$ \\
OBPM-Mercury to HBPM & $4(11 \%)$ & $12(43 \%)$ & $11(39 \%)$ \\
OBPM-Digital to HBPM & $6(21 \%)$ & $7(25 \%)$ & $8(29 \%)$ \\
\hline
\end{tabular}

Normotensive: Normal blood pressure in both methods, Sustained hypertension: Hypertension in both methods, White coat hypertension: Hypertension in first method but normal blood pressure in second one, Masked hypertension: Normal blood pressure in first method but hypertension in second one.

ABPM: Ambulatory blood pressure monitoring, OBPM-Mercury: office blood pressure measurement with mercury sphygmomanometer, OBPMDigital: office blood pressure measurement with digital sphygmomanometer, HBPM: home blood pressure monitoring.

Table 5. Diagnostic efficacy of HBPM over ABPM in hypertension phenotypes detection

\begin{tabular}{|c|c|c|c|c|c|}
\hline Hypertension phenotypes & Sensitivity & Specificity & Positive predictive value & Negative predictive value & Diagnostic accuracy \\
\hline Sustained & $80 \%$ & $65 \%$ & $33 \%$ & $94 \%$ & $68 \%$ \\
\hline White coat & $56 \%$ & $90 \%$ & $91 \%$ & $53 \%$ & $68 \%$ \\
\hline Masked & $100 \%$ & $96 \%$ & $50 \%$ & $100 \%$ & $97 \%$ \\
\hline
\end{tabular}

ABPM: Ambulatory blood pressure monitoring, HBPM: home blood pressure monitoring. 
countries, one of the most important limitations in using out-of-clinic blood pressure measurement methods is the limited access to such methods attributed to economic problems and health systems' lack of coverage. Therefore, to overcome these problems, it is necessary to consider the likelihood of purchasing appropriate equipment, which can increase their utilization. Strong support from the health systems is also needed to increase the utilization of HBPM and allow physicians and healthcare professionals to spend more time educating patients. However, it is worth noting that HBPM results can be misleading when measurement devices are not properly calibrated, or the cuff is not properly set on the right site. In addition, HBPM may cause anxiety and lead to multiple monitoring of blood pressure in patients. There is a risk of change in the process of treatment by the patient without consulting a physician $(10,22)$.

\section{Conclusion}

The findings of the present study showed the frequency of hypertension diagnosed by HBPM was higher than that diagnosed by ABPM; HBPM method had a higher level of diagnostic accuracy than OBPM in diagnosing hypertension. HBPM was able to detect $\mathrm{MH}$ with high diagnostic accuracy. In addition, in more than two-thirds of cases, it was also able to detect $\mathrm{WCH}$ and diagnose patients with sustained hypertension.

\section{Limitations of the study}

Our study had some limitations including, a small sample size and single-center study. Therefore, we suggest that to make general use of HBPM, more studies should be conducted with larger sample size, in multiple centers, and different ethnicities and cultures of our country. Finally, following patient acceptance, our guidelines for diagnosis and treatment of hypertension need to be revised.

\section{Authors' contribution}

All authors passed four criteria for authorship contribution based on recommendations of the International Committee of Medical Journal Editors. SH and NR designed the protocol of the study. NR and SJ developed the protocol and performed it. Analysis of data performed by NR. All authors performed critical revision of the manuscript for important intellectual content, and all of them read and approved the final paper.

\section{Conflicts of interest}

The authors declare that they have no conflicts of interest.

\section{Ethical issues}

The research followed the tenets of the Declaration of Helsinki. The study was also approved by the ethics committee of Qazvin University of Medical Sciences (\#IR.QUMS.REC.1397.134). The institutional ethical committee at Qazvin University of Medical Sciences approved all study protocols. Accordingly, written informed consent was taken from all participants before any intervention. Moreover, ethical issues (including plagiarism, data fabrication, double publication) have been completely observed by the authors.

\section{Funding/Support}

This study was supported by Qazvin University of Medical Sciences (Grant \#14003359).

\section{References}

1. World Health Organization. Global status report on noncommunicable diseases 2010. Geneva: World Health Organization; 2011

2. Shimbo D, Abdalla M, Falzon L, Townsend RR, Muntner P. Role of Ambulatory and Home Blood Pressure Monitoring in Clinical Practice: A Narrative Review. Ann Intern Med. 2015;163:691-700. doi: 10.7326/m15-1270.

3. O'Brien E, Asmar R, Beilin L, Imai Y, Mallion JM, Mancia G, et al. European Society of Hypertension recommendations for conventional, ambulatory and home blood pressure measurement. J Hypertens. 2003;21:821-48. doi: $\quad 10.1097 / 00004872-200305000-00001$.

4. Imai $\mathrm{Y}$, Obara $\mathrm{T}$, Asamaya $\mathrm{K}$, Ohkubo $\mathrm{T}$. The reason why home blood pressure measurements are preferred over clinic or ambulatory blood pressure in Japan. Hypertens Res. 2013;36:661-72. doi: 10.1038/hr.2013.38

5. Cohen JB, Lotito MJ, Trivedi UK, Denker MG, Cohen DL, Townsend RR. Cardiovascular Events and Mortality in White Coat Hypertension: A Systematic Review and Metaanalysis. Ann Intern Med. 2019;170:853-62. doi: 10.7326/ m19-0223

6. Pierdomenico SD, Pierdomenico AM, Coccina F, Clement DL, De Buyzere ML, De Bacquer DA, et al. Prognostic Value of Masked Uncontrolled Hypertension. Hypertension 2018;72:862-69. doi: 10.1161/hypertensionaha.118.11499.

7. Williams B, Mancia G, Spiering W, Agabiti Rosei E, Azizi M, Burnier M, et al. 2018 Practice Guidelines for the management of arterial hypertension of the European Society of Cardiology and the European Society of Hypertension. Blood Press. 2018;27:314-40. doi: $10.1080 / 08037051.2018 .1527177$.

8. Siu AL. Screening for high blood pressure in adults: U.S. Preventive Services Task Force recommendation statement. Ann Intern Med. 2015;163:778-86. doi: 10.7326/m15-2223.

9. Agarwal R, Bills JE, Hecht TJ, Light RP. Role of home blood pressure monitoring in overcoming therapeutic inertia and improving hypertension control: a systematic review and meta-analysis. Hypertension. 2011;57:29-38. doi: 10.1161/ hypertensionaha.110.160911.

10. George J, MacDonald T. Home blood pressure monitoring. Eur Cardiol 2015;10:95-101. doi: 10.15420/ecr.2015.10.2.95.

11. Wood S, Greenfield SM, Sayeed Haque M, Martin U, Gill PS, Mant J, et al. Influence of ethnicity on acceptability of method of blood pressure monitoring: a cross-sectional study in primary care. Br J Gen Pract 2016;66:e577-86. doi: 10.3399/bjgp16X685717.

12. Stergiou GS, Bliziotis IA. Home blood pressure monitoring in the diagnosis and treatment of hypertension: a systematic review. Am J Hypertens. 2011;24:123-34. doi: 10.1038/ ajh.2010.194. 
13. Kang YY, Li Y, Huang QF, Song J, Shan XL, Dou Y, et al. Accuracy of home versus ambulatory blood pressure monitoring in the diagnosis of white-coat and masked hypertension. J Hypertens. 2015;33:1580-7. doi: 10.1097/ hjh.0000000000000596.

14. Rhee MY, Kim SW, Choi EH, Kim JH, Nah DY, Shin SJ, et al. Prevalence of masked hypertension: a population-based survey in a large city by using 24-hour ambulatory blood pressure monitoring. Korean Circ J. 2016;46:681-87. doi: 10.4070/kcj.2016.46.5.681.

15. Nasothimiou EG, Tzamouranis D, Rarra V, Roussias LG, Stergiou GS. Diagnostic accuracy of home vs. ambulatory blood pressure monitoring in untreated and treated hypertension. Hypertens Res. 2012;35:750-5. doi: 10.1038/ hr.2012.19.

16. O’Brien E, Parati G, Stergiou G, Asmar R, Beilin L, Bilo $\mathrm{G}$, et al. European Society of Hypertension position paper on ambulatory blood pressure monitoring. J Hypertens 2013;31:1731-68. doi: 10.1097/HJH.0b013e328363e964. Stergiou GS, Parati G, McManus RJ, Head GA, Myers MG. Guidelines for blood pressure measurement: development over 30 years. J Clin Hypertens (Greenwich). 2018;20:108991. doi: $10.1111 /$ jch.13295.
17. Sharman JE, Howes FS, Head GA, McGrath BP, Stowasser M, Schlaich M, et al. Home blood pressure monitoring: Australian Expert Consensus Statement. J Hypertens. 2015;33:1721-8. doi: 10.1097/hjh.0000000000000673.

18. Parati G, Stergiou GS, Asmar R, Bilo G, de Leeuw P, Imai $\mathrm{Y}$, et al. European Society of Hypertension guidelines for blood pressure monitoring at home: a summary report of the Second International Consensus Conference on Home Blood Pressure Monitoring. J Hypertens. 2008;26:1505-26. doi: 10.1097/HJH.0b013e328308da66.

19. Zhang L, Li Y, Wei FF, Thijs L, Kang YY, Wang S, et al. Strategies for classifying patients based on office, home, and ambulatory blood pressure measurement. Hypertension 2015;65:1258-65. doi: 10.1161/hypertensionaha.114.05038

20. Wagner S. Blood Pressure Self-Measurement. Adv Exp Med Biol 2017;956:97-107.doi: 10.1007/5584_2016_151

21. Villar R, Sánchez RA, Boggia J, Peñaherrera E, Lopez J, Barroso WS, et al. Recommendations for home blood pressure monitoring in Latin American countries: A Latin American Society of Hypertension position paper. J Clin Hypertens (Greenwich) 2020;22:544-54. doi: 10.1111/ jch.13815

Copyright $\odot 2021$ The Author(s); Published by Nickan Research Institute. This is an open-access article distributed under the terms of the Creative Commons Attribution License (http://creativecommons.org/licenses/by/4.0), which permits unrestricted use, distribution, and reproduction in any medium, provided the original work is properly cited. 